\title{
Design of System for Classification of Vocal Cord/Glottis Carcinoma using ANN and Support Vector Machine
}

\author{
Syed Mohammad Ali \\ Department of Electronics \& Telecommunication \\ Engineering, Anjuman College of Engineering \& \\ Technology, Nagpur, India
}

\author{
Pradeep Tulshiram Karule, PhD \\ Department of Electronics Engineering, \\ Yeshwantrao Chavan College of Engineering, \\ Wanadongri, Nagpur, India.
}

\begin{abstract}
Decision support system in voice disorder classification has developed more and more momentum now days because of complication in routine methods. Neurological disorder creates speech problems. Therefore, decision support system can serve as an important mean to detect voice disorders.

In this research work, normal \& vocal cord cancer voice samples are used \& a system is designed to classify vocal cord cancer speech from Normal speech. Vocal cord carcinoma is defined as a malignant tumor in the vocal fold. It is a form of laryngeal cancer, also called as glottis cancer. Pre-processed diseased and normal speech signals are used for spectral analysis to detect disease. Autocorrelation of speech signals is calculated to see the difference between normal and vocal cord cancer speech signal. Two sets of twenty five features are calculated and three neural networks like MLP, GFF, Modular and SVM are used for classification. Feature sets, Networks with highest classification accuracy were found. It is observed that the accuracy of this disease classification is $100 \%$.
\end{abstract}

\section{General Terms}

Biomedical signal processing, Classification, Algorithms.

\section{Keywords}

Vocal cord speech signals; Spectral analysis; Feature extraction, SVM; MLP; Feed forward; Modular Networks.

\section{INTRODUCTION}

Pathological speech detection has received great drive in the last decade. Digital signal processing has become a vital means for speech disorder detection [1]. Due to nature of jobs, harmful societal habits people are subjected to risk of voice problem [2].Usually aged patients are affected by vocal cord cancer. The vocal cord carcinoma leads to early roughness in speech. German voice disorder database and samples from Dr. Naresh Agarwal hospital forms source for this experiment. In this disorder database, patients have pronounced vowel like 'a' [3]. Physicians often use endoscopy to diagnose symptoms of vocal cord carcinoma, however; it is possible to detect disease using definite features of speech signal [1].voice signal is sinusoidal signal having different frequency, amplitude, \& phase. [4].Composition of various organs forms speech signal.

- $\quad$ Lungs, Bronchi, Tracheas producing running out air steam

- Larynx is amplifying the initial speech

- Root of the tongue, throat, nasal cavity, oral cavity forms tone quality \& speech sound [5].
The use of automation techniques to evaluate the larynx and vocal tract helps the speech specialists to perform accurate diagnosis [6].Speech signal in non-intrusive in nature \& it has potential for providing quantitative data with reasonable analysis time and hence study of pathological voice signal has become an significant subject for research as it reduces efforts in diagnoses of disease [7].

Prominent researches in literature survey suggest that most of the research has classified two classes which are normal and pathological disease. Some have worked on dysarthria. Some have classified normal, bicyclic and rough voice or normal, hyper function and paralysis speech. Still, there is an ample scope for suggesting a novel and systematic approach with a view to design an optimal decision support system for diagnosis of dreaded diseases. It is evident that there are many issues, diseases which are yet to be classified and in our work we shall shed light on all these aspects. The algorithm shown in figure 1 below shows the flowchart of the design. Here in this work, speech samples of vocal cord cancer disorder and normal persons were used. These speech samples are passed through pre-emphasis filter which is a high pass filter, the filtered output is framed and then each frame is passed through window. The output signal which is framed and windowed is used for spectral analysis. In spectral analysis derivative of logarithmic spectrum is taken, then logarithmic spectrum is used to get cepstrum. Autocorrelation of speech signal is also found to differentiate normal and vocal cord speech signal. From spectrum, cepstrum and autocorrelation, pattern classification is done for finding normal and vocal cord cancer speech signal.

\section{ALGORITHM}

Algorithm of vocal cord cancer normal speech classification consists of speech acquirement, preprocessing, spectral analysis, feature extraction and disease classification. Speech samples are sinusoidal in nature with different frequency, different amplitude and different phase.

\subsection{Pre-emphasis filter design}

The pre-emphasis filter is high pass filter. This filter flattens the speech signal spectrum and amplifies the area of spectrum.

Thus improving the efficiency of spectral analysis $[8,9]$. The time domain presentation of this filter is represented by the difference equation given below.

$$
Y(n)=X(n)-\lambda X(n-1)
$$




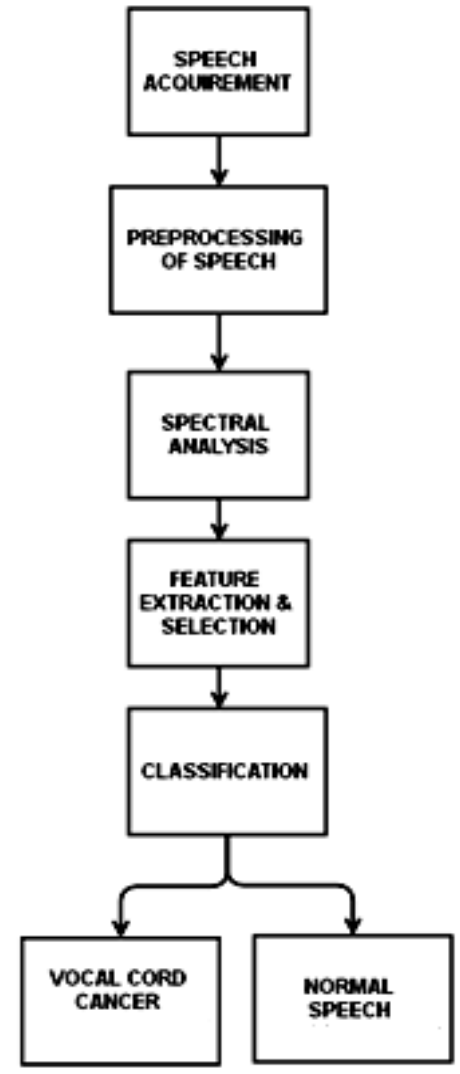

Fig 1: Algorithm of classification

Where $y(n)$ is the output, $x(n)$ is input speech sample $\& \lambda$ is the filter coefficient with $\lambda=0.9375$ optimum result of filtering is received [10]. The filtered and framed output is passed through window. This is done as speech signals are analyzed for short period of time ( $5 \mathrm{msec}$ to $100 \mathrm{msec}$ ). The signal is fairly stationary for shorter period of time [11].

\section{SPECTRAL ANALYSIS AND \\ AUTOCORRELATION}

\subsection{Cepstrum and Derivative of Spectrum}

'Cepstrum' word has been derived by using the first four letter of spectrum. This is a trustworthy way of obtaining dominant fundamental frequency for long clean stationary speech signal. Fourier analysis of the logarithmic amplitude spectrum of the signal is called cepstrum. If the log amplitude spectrum contains several frequently spaced harmonics, then Fourier analysis of the spectrum will confirm a peak matching to the spacing between the harmonics i.e. fundamental frequency. The name cepstrum has come because it turns the spectrum inside out. The $\mathrm{X}$ axis of cepstrum has unit of quefrency \& peak in cepstrum is called rahmonics $[12,13]$.If $\mathrm{X}(\mathrm{n})$ is the speech signal then logarithmic spectrum is given by

$$
\begin{aligned}
& Y(n)=F F T[X(n)] \\
& Y(n)=20 \times \log _{10}[a b s Y(n)]
\end{aligned}
$$

The cepstrum is DFT of log spectrum

$$
Y(n)=F F T[\log (a b s(Y(n)))]
$$

Figure $2 \& 3$ show classification of normal and vocal cord cancer speech using spectrum and cepstrum

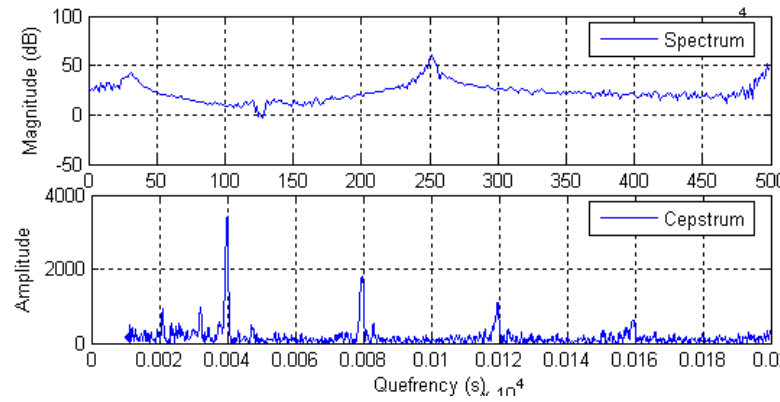

Fig 2: The figures show spectrum and cepstrum of normal person

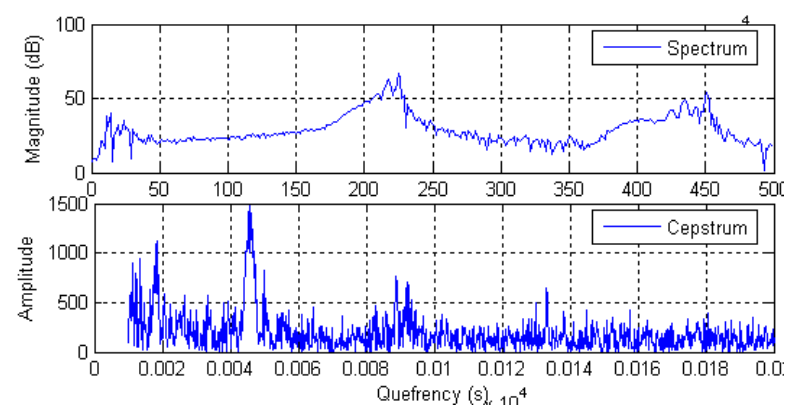

Fig 3: The figures show spectrum and cepstrum of cancer person

\subsection{Autocorrelation of speech signal}

One of the other time domain methods, which is applied for the classification of pathological speech signal from normal is Autocorrelation method. Using this method, one can easily classify the normal and vocal cord cancer patient speech signals. The autocorrelation of discrete time signal $\mathrm{X}(\mathrm{n})$, is given by [4].

$\mathrm{r}_{\mathrm{xx}}(\mathrm{l})=\sum_{\mathrm{n}=-\infty}^{\infty} \mathrm{X}(\mathrm{n}) \cdot \mathrm{X}(\mathrm{n}-\mathrm{l}) \quad \mathrm{l}=0, \pm 1, \pm 2, \ldots$

The autocorrelation function of a signal is a transformation of signal, which is helpful for displaying structure in the waveform [15]. Autocorrelation function is used for calculation of pitch and maximum autocorrelation of frame forms one of the features of this work. Figure 4 and 5 shows

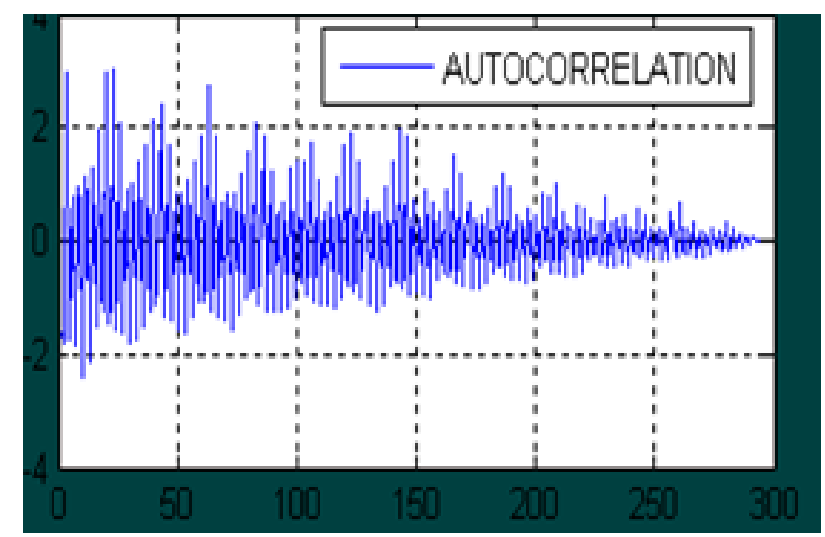

Fig 4: Autocorrelation of normal person 


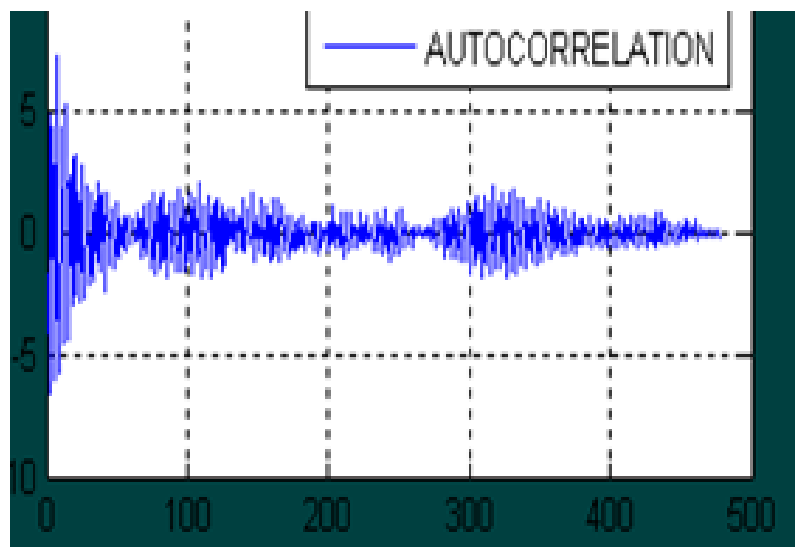

Fig 5: Autocorrelation of vocal cord cancer patient

How the autocorrelation function classifies the speech signals. For the normal signal, the decay of autocorrelation of signal with respect to time is exponential whereas for abnormal decay will not be exponential.

\subsection{Spectrogram}

The spectrogram technique is commonly used as it allows visualization of variation of energy, of the signal as function of both time and frequency [14]. The study investigates the use of the global energy of the signal estimated through spectrogram as a tool for discrimination between signals obtained from healthy and pathological subjects as shown in figure $6 \& 7$ [13].

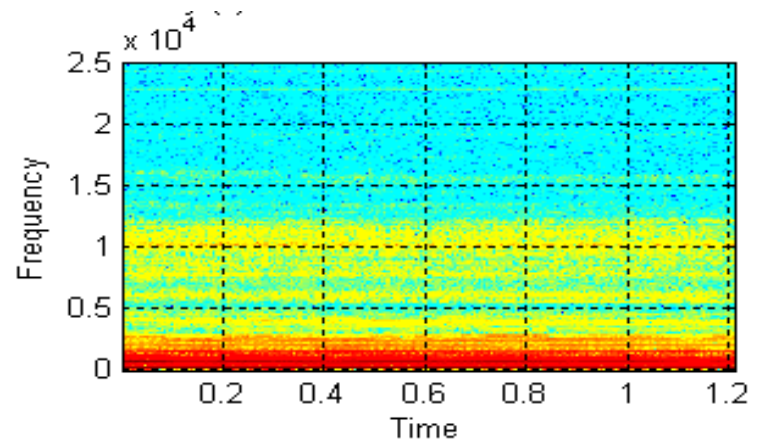

Fig 6: The above figures show spectrogram of normal person

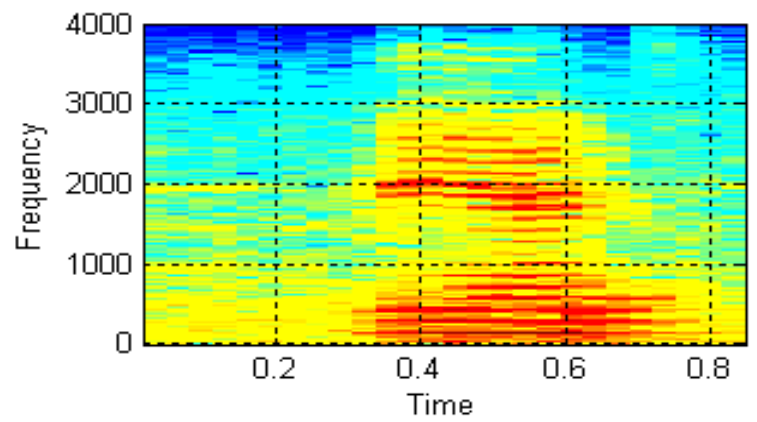

Fig 7: The above figures show spectrogram of vocal cord cancer person

\section{FEATURE EXTRACTION FEATURE SELECTION}

Two sets of feature i.e. set- 1 and set- 2 are shown in the table 1 $\& 2$ respectively. The expressions for features which has been calculated are given below.

Table 1. Feature set-1

\begin{tabular}{|c|c|c|}
\hline Sr No. & Feature set-1 & Number of Features \\
\hline 1. & $\begin{array}{c}\text { Mel frequency Cepstrum } \\
\text { coefficients (MFCC) }\end{array}$ & 06 \\
\hline 2. & Delta coefficient & 03 \\
\hline 3. & $\begin{array}{c}\text { Delta-Delta coefficient } \\
\text { Linear Predictive Cepstral } \\
\text { coefficients }\end{array}$ & 08 \\
\hline 4. & $\begin{array}{c}\text { Formant frequencies } \\
\text { Pitch }\end{array}$ \\
\hline 6. & Total & 04 \\
\hline & \multicolumn{2}{c}{} \\
\hline
\end{tabular}

Table 2. Feature set-2

\begin{tabular}{|c|c|c|}
\hline Sr. No. & Feature set- 2 & Number of Features \\
\hline 1. & $\begin{array}{l}\text { Mel frequency Cepstrum } \\
\text { coefficients (MFCC) }\end{array}$ & 01 \\
\hline 2. & LPCC & 01 \\
\hline 3. & Formant Frequencies & 04 \\
\hline 4. & Pitch & 01 \\
\hline 5. & Lpc & 01 \\
\hline 6. & Spectral flux & 01 \\
\hline 7. & Spectral Centroid & 01 \\
\hline 8 & Spectral Decrease & 01 \\
\hline 9. & Spectral Crest & 01 \\
\hline 10 & Spectral Roll off & 01 \\
\hline 11 & Entropies & 05 \\
\hline 12. & Short Time Energy & 01 \\
\hline 13. & ZCR & 01 \\
\hline 14 & Peak value & 01 \\
\hline 15 & RMS value & 01 \\
\hline
\end{tabular}




\begin{tabular}{|c|c|c|}
\hline 16 & Max Autocorrelation & 01 \\
\hline 17 & Standard deviation & 01 \\
\hline 18 & Variance & 01 \\
\hline & Total & 25 \\
\hline
\end{tabular}

\subsection{Pitch calculation}

Pitch detection is an essential task in a variety of speech processing applications. Here we will be proposing cepstrum method and autocorrelation method. Autocorrelation method of calculation of pitch gives optimum result.

\subsection{Formant frequency estimation}

Frequencies of resonance for each frame are called formants. It is measured as an amplitude peak in frequency spectrum of the speech. The system function of LPC filter is given by

$\mathrm{H}(\mathrm{z})=\frac{\mathrm{Y}(\mathrm{z})}{\mathrm{X}(\mathrm{z})}=\frac{1}{1-\sum_{\mathrm{k}=1}^{\mathrm{p}} \mathrm{a}_{\mathrm{k}} \mathrm{z}^{-\mathrm{k}}}=\frac{1}{\mathrm{~A}(\mathrm{z})}$

\subsection{Short Time Energy (STE)}

It is known that diseased speech signal has greater amplitude variations as compared normal speech signal; here short time energy has been used to counter the amplitude variation in diseased speech signal. The short time energy is expressed as

$E_{\hat{n}}=\sum_{m=-\infty}^{\infty}(x[m] w[\hat{n}-m])^{2}$

\subsection{Short Time Zero Crossing Rate (ZCR)}

Short time ZCR is defined as the number of times the speech signal changes sign within a given window. The ZCR in case of stationary signal is defined as,

$\left.\mathrm{Z}_{\widehat{\mathrm{n}}}=\sum_{\mathrm{m}=-\infty}^{\infty}(0.5|\operatorname{sgn}\{\mathrm{x}[\mathrm{m}]\}-\operatorname{sgn}\{\mathrm{x}[\mathrm{m}-1]\}| \mathrm{w}[\hat{\mathrm{n}}-\mathrm{m}])\right)$

Where, $\operatorname{sgn}\{x\}=\left\{\begin{array}{cc}1 & x \geq 0 \\ -1 & x<0\end{array}\right.$

\subsection{Spectral Centroid (SC)}

Weighted average of the frequency of the spectrum is called as spectral centroid. and thus would give us an idea as to what frequency range most of the power of spectrum would lie in. The spectral centroid is given by

$\mathrm{SC}=\sum_{\mathrm{k}=0}^{\mathrm{N}-1} \mathrm{X}(\mathrm{k}) \mathrm{F}(\mathrm{k}) / \sum_{\mathrm{k}=0}^{\mathrm{N}-1} \mathrm{X}(\mathrm{k})$

Where $X(k)$ represents weighted frequency value or magnitude of bin number $\mathrm{k}, \mathrm{F}(\mathrm{k})$ represents center frequency of that bin.

\subsection{Spectral Flux (SF)}

The spectral flux is defined as the difference in the power spectra of two consecutive speech frames.

$\mathrm{F}_{\mathrm{r}}=\sum_{\mathrm{k}=1}^{\mathrm{N} / 2}\left(\left|\mathrm{X}_{\mathrm{r}}[\mathrm{k}]\right|-\left|\mathrm{X}_{\mathrm{r}-1}[\mathrm{k}]\right|\right)^{2}$

\subsection{Mel Frequency Cepstral Coefficient (MFCC)}

Frame the signal into short frames. For each frame calculate the period gram estimate of power spectrum. Apply the Mel filter bank to power spectra, sum the energy in each filter. Take the logarithm of all filter bank energies; Take the DCT of $\log$ filter bank energies. DFT for $i^{\text {th }}$ frame is given by

$$
\begin{array}{r}
\mathrm{x}_{\mathrm{i}}(\mathrm{k})=\sum_{\mathrm{n}=1}^{\mathrm{N}} \mathrm{x}_{\mathrm{i}}(\mathrm{n}) \mathrm{h}(\mathrm{n}) \mathrm{e}^{-\mathrm{j} 2 \pi \mathrm{kn} / \mathrm{N}} \\
1 \leq k \leq K
\end{array}
$$

Where, $\mathrm{h}(\mathrm{n})$ is hamming window and $\mathrm{k}$ is the length of DFT

Power spectrum of $i^{\text {th }}$ frame is given by

$P_{i}(k)=\frac{1}{N}\left|x_{i}(k)\right|^{2}$

Delta and delta-delta coefficients are obtained by recursive formula.

\subsection{Time Envelope}

Another possibility for the above mentioned smoothing is to take the maximum of the absolute amplitude values in each frame. The result is an envelope trajectory that lies on the peaks of the time-domain signal:

$\left.E N V_{r}=\max \operatorname{Xr}[n] \mid\right\}$

$$
n=1, N
$$

\subsection{Root Mean Square}

One possible way to perform this smoothing is to compute the RMS Energy of the signal in each frame, and it is given by

$R M S=\sqrt{\frac{1}{N+1} \sum_{n=0}^{N}|x[n]|^{2}}$

$\mathrm{N}$ is the number of samples in each analysis window.

\subsection{Spectral Crest}

Spectral crest is defined as ratio of peak value to RMS value of speech signal in each frame.

\subsection{Roll Off}

Here, it is defined as the frequency below which $85 \%$ of the accumulated magnitudes of the spectrum are concentrated. That is, if $\mathrm{K}$ is the bin that

$\sum_{\mathrm{k}=0}^{\mathrm{M}}\left|\mathrm{X}_{\mathrm{r}}(\mathrm{k})\right|=0.85 \sum_{\mathrm{k}=1}^{\mathrm{N} / 2}\left|\mathrm{X}_{\mathrm{r}}(\mathrm{k})\right|$

Fulfils then, the role off is $\mathrm{Rr}=\mathrm{f}[\mathrm{K}]$.

\subsection{Spectral Decrease}

Spectral decrease estimates the steepness of the decrease of spectral envelop over frequency given by

$\mathrm{V}_{\mathrm{SD}}(\mathrm{n})=\frac{\sum_{\mathrm{k}=1}^{\mathrm{k} / 2-1} \frac{1}{\mathrm{k}}(\mid \mathrm{X}(\mathrm{k}, \mathrm{n}|-| \mathrm{X}(0, \mathrm{n}) \mid)}{\sum_{\mathrm{k}=1}^{\mathrm{k} / 2-1}|\mathrm{X}(\mathrm{k}, \mathrm{n})|}$

\subsection{Entropies}

The five entropies which are Sure, Shannon, Threshold Normalized and Log energy have been estimated. 


\subsection{Standard deviation, Variance}

The other features which were used are Standard deviation, Variance, maximum autocorrelation, Lpc.

Every new feature should put some new information about the disease. In this work, in what way these features vary with respect to above disease is seen and if there is variation mean they are giving some information then they were considered. Variation of fo is shown in Fig.8. Each single feature was trained and tested for considered diseases and in this way feature vector was formed. The $\%$ corrects detection of feature fo for chordectomy, cancer, laryngitis, laryngeal paralysis and psychogenic dysphonie diseases are $0,0,78.26,29.41,88.23$ respectively. This means that feature fo is good at accuracy for laryngitis and psychogenic dysphonie disease. So we have used fo as one of the features in feature vector.And in this way all the features were tested and those features with good accuracy were considered.

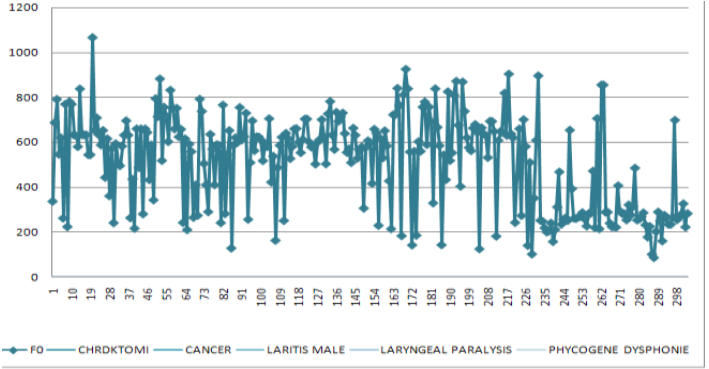

Fig 8: The above figures show spectrogram of vocal cord cancer person

\section{CLASSIFICATION}

The classifiers which were used for classification are MLP, GFF, Modular and Support Vector Machine (SVM). Two sets of twenty five features were chosen for classification. In the first three N/Ns, five transfer functions with single layer network, which were used are Tanh axion, L-Tanh axion, Sigmoid axion, L-sigmoid axion and Softmax axion were used and in SVM only epochs can be changed so we have changed the epochs. In this case male samples of vocal cord cancer and normal were taken, as males are ten times prone to this disease. Training was done for $75 \%$ of samples for three runs and testing on $25 \%$ of samples. The total number of samples taken are 106. All the experimental values put in tabular form are given in table 3 . It is clear from all the experiment i.e $1 \mathrm{~A}, 1 \mathrm{~B}, 2 \mathrm{~A}, 2 \mathrm{~B}, 3 \mathrm{~A}, 3 \mathrm{~B}, 4 \mathrm{~A}$ and $4 \mathrm{~B}$ that the detection of vocal cord cancer disease and normal from the speech is $100 \%$.

\section{RESULT AND DISCUSSION}

We have performed four experiments and the neural networks were used are MLP, GFF, MODULAR and SVM and for each network, we have used two sets of feature i.e. set- 1 and set- 2 . In the first three experiments we have used five transfer function which are Tanh axion, Sigmoid axion, Linear tanhaxion, Linear sigmoid axion and softmax axion, epochs used were 1500 and for last experiment epochs used were $1500 \& 2000$. Graph 1 is the graph of accuracy of MLP verses transfer function from which we can conclude that for feature set-1, except for softmax axion, the accuracy is $100 \%$ in determining the vocal cord cancer disease from normal for all other four specified transfer functions. And similarly in the same experiment with feature set-2, except for Tanh axion, the accuracy is $100 \%$ in determining the vocal cord cancer disease from normal for other specified transfer function.
The second experiment is of classification with GFF $\mathrm{n} / \mathrm{w}$ and from graph 2 , we conclude that, with set- 1 as feature, the accuracy is $100 \%$ for all the five transfer function and with set -2 as feature vector, the accuracy is $100 \%$ only for Linear Tanh axion.

In experiment three where classification is done with modular $\mathrm{n} / \mathrm{w}$, from graph 3 , with set-1 as features and Tanhaxion, Linear Tanhaxion, and Softmaxaxion as transfer function we conclude that the accuracy is $100 \%$. And with set -2 as feature accuracy is $100 \%$ only for Linear Tanh axion.

Table 3. Experimental values and Accuracy

\begin{tabular}{|c|c|c|c|c|}
\hline $\begin{array}{l}\mathbf{S} \\
\mathbf{r} \\
\dot{\mathbf{N}} \\
\mathbf{0}\end{array}$ & $\begin{array}{c}\text { Experiment- } \\
\text { ation, } \\
\text { N/N, } \\
\text { Feature sets }\end{array}$ & $\begin{array}{l}\text { Transfer } \\
\text { Function }\end{array}$ & Epoch & $\begin{array}{l}\text { Maximum } \\
\% \\
\text { Accuracy }\end{array}$ \\
\hline \multirow{5}{*}{1} & \multirow{5}{*}{$\begin{array}{l}\text { 1A, MLP, } \\
\text { set-1 }\end{array}$} & Tanh Axion & 1500 & 100 \\
\hline & & Sigmoid Axion & 1500 & 100 \\
\hline & & $\begin{array}{c}\text { Linear } \\
\text { TanhAxion }\end{array}$ & 1500 & 100 \\
\hline & & $\begin{array}{c}\text { Linear Sigmoid } \\
\text { Axion }\end{array}$ & 1500 & 100 \\
\hline & & Softmax Axion & 1500 & 92.30 \\
\hline \multirow{5}{*}{2} & \multirow{5}{*}{$\begin{array}{l}\text { 1B, MLP, } \\
\text { set-2 }\end{array}$} & Tanh Axion & 1500 & 92.30 \\
\hline & & Sigmoid Axion & 1500 & 100 \\
\hline & & $\begin{array}{c}\text { Linear } \\
\text { TanhAxion } \\
\end{array}$ & 1500 & 100 \\
\hline & & $\begin{array}{c}\text { Linear Sigmoid } \\
\text { Axion }\end{array}$ & 1500 & 100 \\
\hline & & Softmax Axion & 1500 & 100 \\
\hline \multirow{5}{*}{3} & \multirow{5}{*}{$\begin{array}{l}\text { 2A, GFF, } \\
\text { set-1 }\end{array}$} & Tanh Axion & 1500 & 100 \\
\hline & & Sigmoid Axion & 1500 & 100 \\
\hline & & $\begin{array}{c}\text { Linear Tanh } \\
\text { Axion }\end{array}$ & 1500 & 100 \\
\hline & & $\begin{array}{c}\text { Linear Sigmoid } \\
\text { Axion }\end{array}$ & 1500 & 100 \\
\hline & & Softmax Axion & 1500 & 100 \\
\hline \multirow{5}{*}{4} & \multirow{5}{*}{$\begin{array}{l}\text { 2B, GFF, } \\
\text { set-2 }\end{array}$} & Tanh Axion & 1500 & 92.30 \\
\hline & & Sigmoid Axion & 1500 & 92.30 \\
\hline & & $\begin{array}{c}\text { Linear Tanh } \\
\text { Axion }\end{array}$ & 1500 & 100 \\
\hline & & $\begin{array}{c}\text { Linear Sigmoid } \\
\text { Axion }\end{array}$ & 1500 & 92.30 \\
\hline & & Softmax Axion & 1500 & 84.61 \\
\hline \multirow{5}{*}{5} & \multirow{5}{*}{$\begin{array}{l}\text { 3A, Modular, } \\
\text { set-1 }\end{array}$} & Tanh Axion & 1500 & 100 \\
\hline & & Sigmoid Axion & 1500 & 57.69 \\
\hline & & $\begin{array}{l}\text { Linear Tanh } \\
\text { Axion }\end{array}$ & 1500 & 100 \\
\hline & & $\begin{array}{c}\text { Linear Sigmoid } \\
\text { Axion }\end{array}$ & 1500 & 65.38 \\
\hline & & Softmax Axion & 1500 & 100 \\
\hline \multirow{5}{*}{6} & \multirow{5}{*}{$\begin{array}{l}\text { 3B, Modular, } \\
\text { set-2 }\end{array}$} & Tanh Axion & 1500 & 92.30 \\
\hline & & Sigmoid Axion & 1500 & 65.38 \\
\hline & & $\begin{array}{c}\text { Linear } \\
\text { TanhAxion }\end{array}$ & 1500 & 100 \\
\hline & & $\begin{array}{c}\text { Linear Sigmoid } \\
\text { Axion } \\
\end{array}$ & 1500 & 65.38 \\
\hline & & Softmax Axion & 1500 & 88.46 \\
\hline 7 & $\begin{array}{l}\text { 4A, } \text { SVM, } \\
\text { set-1 }\end{array}$ & ------- & 1500 & 100 \\
\hline
\end{tabular}




\begin{tabular}{|c|c|c|c|l|}
\hline $\begin{array}{c}\text { S } \\
\mathbf{r}\end{array}$ & $\begin{array}{c}\text { Experiment- } \\
\text { ation, } \\
\mathbf{N}\end{array}$ & $\begin{array}{c}\text { Transfer } \\
\text { N }\end{array}$ & $\begin{array}{l}\text { Function } \\
\text { opoch }\end{array}$ & $\begin{array}{l}\text { Maximum } \\
\% \\
\text { Accuracy }\end{array}$ \\
\hline 8 & $4 \mathrm{~B}, \mathrm{SVM}$, & ---- & 1500, & $84.61,100$ \\
\hline
\end{tabular}

Fourth experiment is with SVM, in which we can change only epochs. So, in this experiment with set-1 as feature, 1500 epochs, we got $100 \%$ accuracy. And when set- 2 was used as feature vector, with 2000 epochs we got $100 \%$ classification accuracy. The accuracy of this experiment for two different set of feature is shown in bar chart graph 4 .

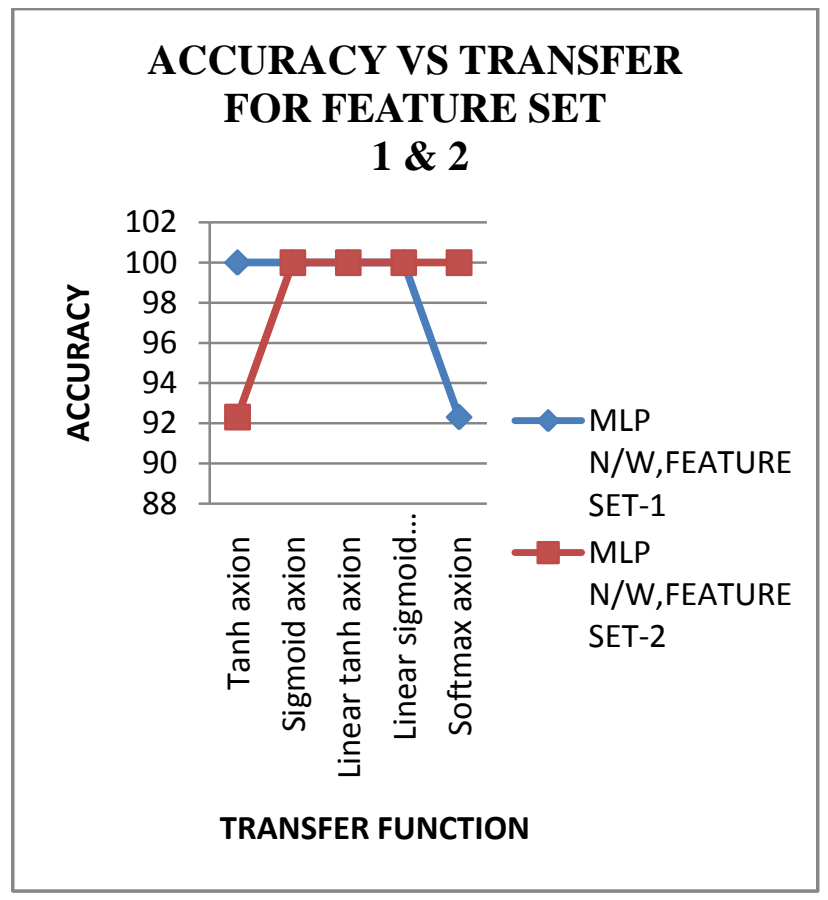

Graph 1: Accuracy verses Transfer function for MLP

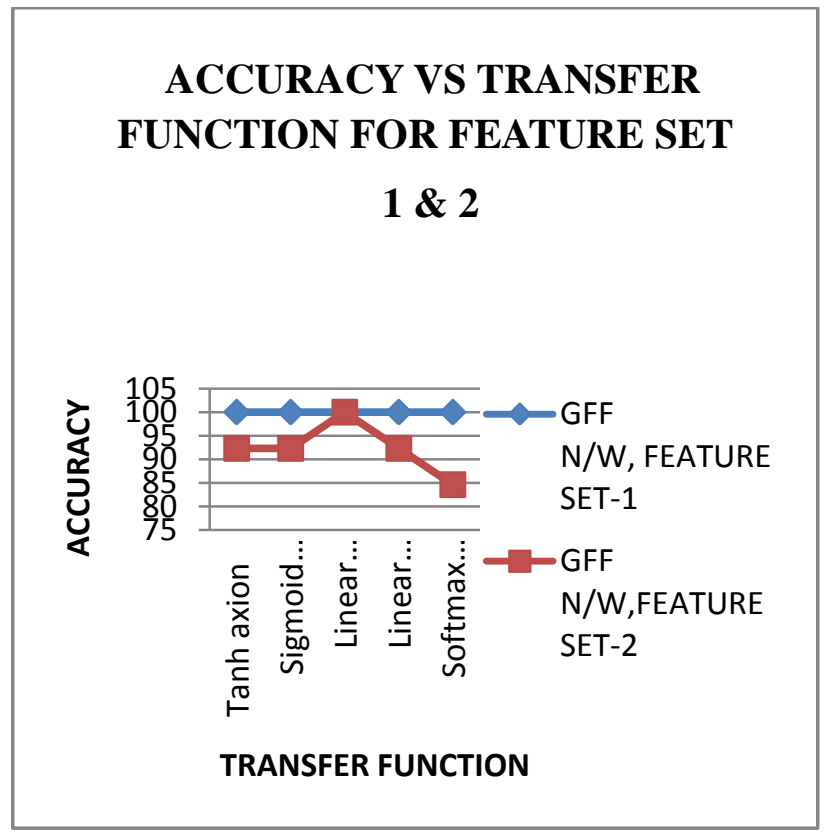

Graph 2: Accuracy verses Transfer function for GFF
Thus, finally from all the four experiments, we can conclude that, mostly accuracy is $100 \%$ with Tanh Axion and Linear Tanh axion as transfer function..In SVM, with $1.5 \mathrm{~K}$ and $2 \mathrm{k}$ epochs the accuracy is $100 \%$ for both the feature sets. The input and output nodes used in the network were Twenty-five and two respectively, since twenty features were used to classify two classes. Future scope for this experiment may be reduced new features to get $100 \%$ accuracy. Hence, for all four networks i.e MLP, GFF, Modular network and SVM, The Vocal cord cancer from Normal speech is $100 \%$ classified.

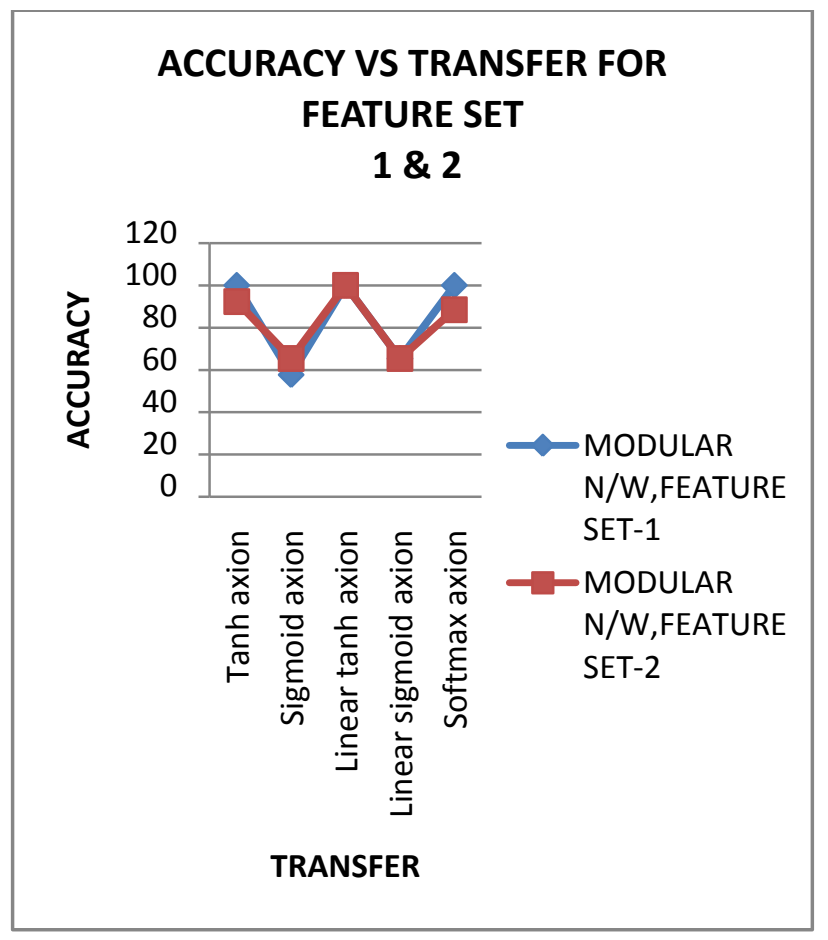

Graph 3: Accuracy verses Transfer function for Modular $\mathbf{n} / \mathbf{w}$

\section{ACCURACY ,FEATURE SET 1 \& 2}

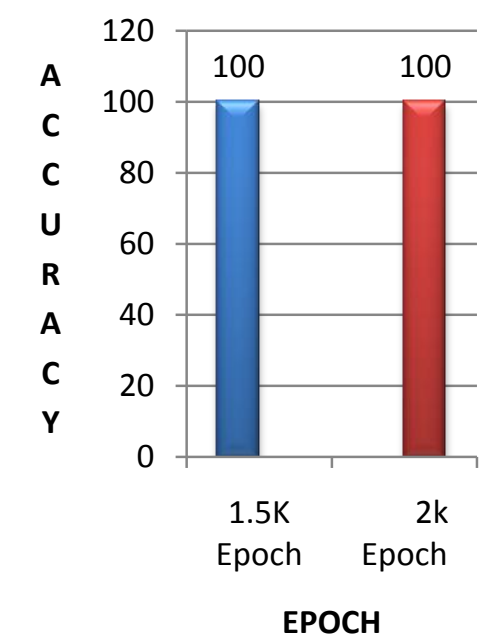

Graph 4 : Accuracy verses Transfer function for SVM 


\section{REFERENCES}

[1] Salhi, L., Mourad, T., Cherif, A.,2010 "Voice Disorders Identification Using Multilayer Neural Network", The International Arab Journal of Information Technology, Volume 7-No.2, (April 2010),177-185.

[2] Hariharan, M., Paulraj, M.P., Jaacob, S., 2010, "Time Domain Features and Probabilistic Neural Network For the Detection Of Vocal Fold Pathology", Malaysian journal Of Computer Science, Vol(23) (2010),60-67.

[3] Putzer, M., Koreman, J., 1997, "A german database for a pattern for vacal fold vibration" Phonus 3, Institute of Phonetics, University of the Saarland, Tavel, P. 2007 Modeling and Simulation Design. AK Peters Ltd, 143153.

[4] Proakis, J. G. G., Manolakis, "Digital Signal Processing. Principles, Algorithm and Applications", Prentice Hall India, Third Eition.309, 122.

[5] Orzechowski, Izworski,A., Izworski, R., Tadeusiewiez ,K., Chmunzynska, P., Radkowski, I., Gotkowska, 2005, " Processing of pathological change in speech caused by Dysarthria ", IEEE Proceedings of 2005 International symposium on intelligent signal processing \& communication system, 49-52.

[6] Oliveira Rosa, M. de, Pereira ,J. C, Gellet, M.,2000 "Adaptive Estimation of Residue Signal for Voice Pathology Diagnose", IEEE Transaction on Biomedical Engineering, Vol.47, No.1(Jan.2000).

[7] Cesar, M. E., Hugo, R. L.,2000, "Acoustic Analysis of speech for detection of Laryngeal pathologies", IEEE proceeding of the 22nd Annual EMBS international conference chicago IL,(2000),2369-2372.
[8] Picone, J.W., 1993 "Signal modeling techniques in speech recognition", Proceedings of the IEEE, Vol.81, No.9, Sept.1993.

[9] Lipeika, A., Lipeikiene, J., Lipeikiene, L., Telksnys,2000, "Development of Isolated speech Recognition System", INFORMATICA, Vol 13, No.1, 2002,37-46

[10] Sigmund, M. "Voice Recognition By Computer", TectumVerlag publication, pp no20-22.

[11] Gill, M. K.,2010 "Vector Quantization based Speaker identification", International Journal of Computer Application(0975-8887) Volume 4-No.2,2010,1-4.

[12] Schafer ,R.W., Rabiner, L.R.,1970 "System for automatic formant analysis of voiced speech,"J.Amer.,vol.47, (Feb.1970) 634-648,

[13] http://www.phon.ucl.ac.uk/resource/sfs/rtgram/AboutSpe ctrography

[14] Fernandes, M., Mattioli, F.E.R., LamounierJr.,E.A. and Andrade,A.O.,2011,“Assesment of Laryngeal Disorders Through TheGlobal Energy of Speech,"IEEE Latin American Transactions,vol.9,No.7,( December 2011).

[15] Rabiner,L. R.,1977 "On the use of Autocorrelation Analysis for Pitch Detection",IEEE Transaction Acoustics,Speech and signal Processing ",Vol.ASSP 25,No.1, (February 1977),24-30. 